\title{
Real-Time Twitter Sentiment toward Midterm Exams
}

\author{
Wei Hu \\ Department of Computer Science, Houghton College, New York, USA \\ Email: wei.hu@houghton.edu
}

Received December $3^{\text {rd }}$, 2011; revised January $7^{\text {th }}$, 2012; accepted February $6^{\text {th }}, 2012$

\begin{abstract}
Twitter is the most popular microblogging service today, with millions of its uers posting short messages (tweets) everyday. This huge amount of user-generated content contains rich factual and subjective information ideal for computational analysis. Current research findings suggest that Twitter data could be utilized to gain accurate public sentiment on various topics and events. With help of Twitter Stream API, we collected 260,749 tweets on the subject of midterm exams from students on Twitter for two consecutive weeks (Oct 17-Oct 30, 2011). Our aim was to investigate the real-time Twitter sentiment on midterm exams by hour, day, and week for these two weeks, using a sentiment predictor built from an opinion lexicon augmented for this specific domain. At different levels of temporal granularity, our analysis revealed the variation of sentiment. The average sentiment of the first week (Oct 17-23) was more negative than the second week (Oct 24-30). For both weeks, the overall trend curves of sentiment increased from Monday to Sunday. For each weekday, there was a period around 9:00 am-5:00 pm EST that had maximum sentimet. On each weekend, the sentiment values during a day reached their maximum between 5:00 am to 8:00 am, and then decreased after 8:00 am. Furthermore, we observed some consistent group behavior of Twitter users based on seemingly random behavior of each individual. The lowest number of tweets always occured around 5:00 am-6:00 am each day, and the maximum number was around 1:00 pm except Sunday. The minimum of tweet lengths happened usually around 9:00 am and the maximum length was around 4:00 am everyday. Twitter users with positive sentiment appeared to have more friends and followers than those carrying negative sentiment. Also, users who shared the same sentiment inclined to have similar ratios of friends and followers, which is not true for general users.
\end{abstract}

Keywords: Twitter; Sentiment; Midterm Exam; Opinion; Lexicon; Social Media

\section{Introduction}

Twitter, founded in 2006, is the most popular microblogging service with millions of users sharing information and opinions everyday. The messages posted on Twitter, termed tweets or updates, are short and limited to 140 characters including punctuaton and spacing, averaging 11 words per message. As a phenomenal online social networking site, Twitter provides an unprecedent rich source of data containing facts and opinions for text mining and analysis, bringing in many new oppornuties and intellectual challenges.

In the field of text mining, there has been a shift from traditional fact based analysis to opinion oriented analysis, i.e., from classifying docments by their topics such as sports, health, or entertainment to their sentiment about a particular subject or event such as a movie or a commercial product. In text classification of documents by topic, there might be many possible categories. In contrast, in sentiment classification there are relatively few classes, say positive or negagtive, that cover many domains. Compared to topic discovery, sentiment is difficult to identify, because it can be expressed in a very subtle manner. Furthermore, sentiment is context sensitive and domain dependent. The same sentence can exhibit opposite sentiments in two different contexts or domains. As a result, one sentiment predictor may perform well in one targeted domain, but may perform poorly in other domains.

Historically there has been extensive research on mining and retrieval of factual information, including Web search, text classification, and text clustering. However, sentiment has emerged as a new subject of research recently because of the explosion of public user-generated content in online social media. Identifying opinions expressed in social media is a popular way of interpreting this type of data, which could lead to a broad range of applications. Companies and organizations can improve their products and services according to the sentiment of their customers. The opinion of one individual might only represent the subjective view of this person, but the collection of opinions from a large number of people are nonetheless statistically significant and influential, and therefore are accurate public indicators of different topics and events.

Sentiment analysis, also known as opinion mining, is the computational extraction of opinion, sentiment, and emotion in text. There is an excellent survey on this subject (Pang, 2008). Sentiment can be analyzied at various levels: document, section, paragraph, and sentence, with document as the most common level. There are studies on general sentiment from standard and long documents, and Twitter specific sentiment (Go et al., 2009; Pak \& Paroubek, 2010). At the document level, the work in (Pang, 2002; Turney, 2002) evaluated the polarity of product reviews and movie reviews respectively.

Statistical natural language processing and machine learning are two commom methods in sentiment analysis. With natural language processing, the opinon polarity of a document or a sentence is determined using a set of indicative opinion words, an opinion lexicon, that express positve or negative sentiment such as "good" or "bad". The machine learning approach is to build a sentiment classifier based on manually labeled training 
data to predict the class of sentiment (positive, negative, or neutral). Obtaining large size of training data annotated by experts is difficult, and sometimes human judgment of the sentiment expressed in text is not as accurate as an automated approach. To overcome these difficulties, recently there were reports that combined both techniques (Lu \&Tsou, 2010; Tan et al., 2008).

Using movie reviews as data, machine learning techniques were found to be more effective in sentiment collection than human produced baselines. But they didn't predict as well on sentiment as on traditional topic based classification, implying that the sentiment classification was more challenging (Pang, 2008). To improve the performance of the machine learning approach in (Pang, 2008), a novel technique of finding minimum cuts in graphs was proposed to extract the subjective portions of the document, thus removing the irrelevant text while keeping the subjective portion (Pang et al., 2002).

Due to the 140 character limitation on tweets, Twitterers have adopted abbreviated and slang expressions to overcome this limit. Thus, they have created a language of different flavor in this social media than the one used in traditional texts. Tweets also contain misspellings, and are shorter and more ambiguous than other sentiment data such as reviews and blogs. Another feature of tweets is that they cover a variaty of topics unlike other blogging sites that are more focused on one or a few topics. Consequently, it is not straightforward to detect the sentiment of tweets.

People typically use Twitter for daily chatter, conversation, information sharing, and news reporting (Java et al., 2007). A study showed that $19 \%$ of tweets mention a certain brand, and $20 \%$ of them contain a sentiment (Jansen et al., 2009). In general, these massages could be classified into two groups: about Twitter users themselves and information sharing. In both cases, tweets contain information about the mood of their writers (MorNaaman \& Boase, 2010). With six dimensions of mood (tension, depression, anger, vigor, fatigue, confusion), the public mood patterns learned from Twitter data were found to be related to real offline events, such as changes in the stock market and the oil price, and the outcome of a political election (Bolle et al., 2011). To find the connection between public opinions from polls and the sentiment from Twitter messages (O’Connor et al., 2010), positive and negative words were defined by a subjectivity lexicon, a set of words containing about 1600 and 1200 words marked as positive and negative, respectively. A message was defined as positive if it contained any positive word, and negative if it contained any negative word. This allowed for messages to be both positive and negative. The results from (O’Connor et al., 2010) showed that the sentiment from Twitter data was highly correlated with the polls.

Sentiment detection of tweets is one of the fundemental components in the applications using Twitter data. There are several sentiment tools developed for Twitter data such as Twendz, Twitter Sentiment, TweetFeel, and Viralheat, but most of them are still lacking the expected accuracy due to the unique characteristics of tweets.

In this report, we sought to examine a stream of text messages from students on Twitter to gather real-time sentiment toward midterm exams. Our main interest was to discover the fluctuation in sentiment about midterms from this particular group of Twitter users by hour, day and week, thus our investigation could disclose the sentiment at different levels of temporal granularity. Twiter Stream API made it possible to analyze sentiment for this topic as they arose in real-time.
Though many colleges allow students to evaluate their courses, Twitter provides a venue for them to express opinions on their midterm exams. Students have different feelings about the midterm exams. They can have high confidence in the coming exams because they have studied and prepared well, otherwise, they may feel uncertain, uneasy, afraid, scared, and anxious. They also can express the feelings to their teachers, exams in general, and their grades from these exams.

\section{Materials and Methods}

\section{Twitter Data}

Twitter is a service for information network and communication, which produces more than 200 million tweets a day. Twitter offers three APIs to access its corpus of data and support developers to build applications using Twitter data. The Search API allows a user to query for Twitter content, and the REST API enables the access to some of the core primitives of Twitter including timelines, status updates, and user information. Finally, the Streaming API is the real-time sample of the Twitter Firehose with a long-lived HTTP connection to retrieve tweets by user ids, keywords, random sampling, geographic location, etc. This API is best for building data mining applications.

Using Twitter Stream API (https://dev.twitter.com/docs/strea ming-api) and Twitter4J (http://twitter4j.org), we collected a corpus of 260,749 tweets on midterm exmas during a period of two consecutive weeks, from Oct 17 to Oct 30, 2011. The detailed information for the numbers of tweets collected by day is presented in Table 1.

To gain a preliminary view of our tweet data, we calculated the average tweet count and tweet length by hour during these two weeks (Figure 1). Amazingly, some group patterns of these student Twitters were detected from the random behavior of each individual. The lowest average tweet count was always around 5:00 am-6:00 am each day, and the maximum count was around 1:00 pm except Sunday. Remarkably, the minimum average length was regularly around 9:00 am and the maximum length was around 4:00 am.

\section{Sentiment Predictor}

In the present study, we employed an opinion lexicon ( $\mathrm{Hu} \&$ Bing, 2004) of around 6800 words to build our sentiment predictor. Several opinion lexicons exist, but a web derived lexicon like the one from (Hu \& Bing, 2004) could improve lexicon-based sentiment evaluation (Velikovich et al., 2010).

Considering the nature of midterm exams, we augmented the opinio lexicon from (Hu \& Bing, 2004) with some domain specific words such as "bombed", and "aced", and removed some negative words such as "criminal", "fall”, and "break" from this lexicon since "criminal" in our context can be part of the name of an exam like "criminal justice midterm", "fall" could mean fall semester, and "break" could mean a college break that students look forward to.

Encouraged by the results in (O’Connor et al., 2010), we adopted their approch in our study to count instances of positive and negative words and emoticons, when evaluating the sentiment of a tweet on midterms using an opinion lexicon. Considering the characteristics of tweets, a weight +1 was assgined to a positive word, -1 to a negative word, +5 to a positive emoticon, and -5 to a negative emoticon, since emoticons are key non-verbal sentiment indicators in tweets. Furthermore, we 
Table 1.

Number of tweets collected by day from Oct 17 to Oct 30, 2011.

\begin{tabular}{cccccccccc}
\hline & Monday & Tuesday & Wednesday & Thursday & Friday & Saturday & Sunday & total \\
\hline Week1 (Oct 17-23) & 27652 & 30660 & 31222 & 29147 & 15095 & 5072 & 9411 & 148259 \\
Week2 (Oct 24-30) & 24880 & 24474 & 23257 & 19377 & 10779 & 3761 & 5962 & 112490 \\
\hline
\end{tabular}
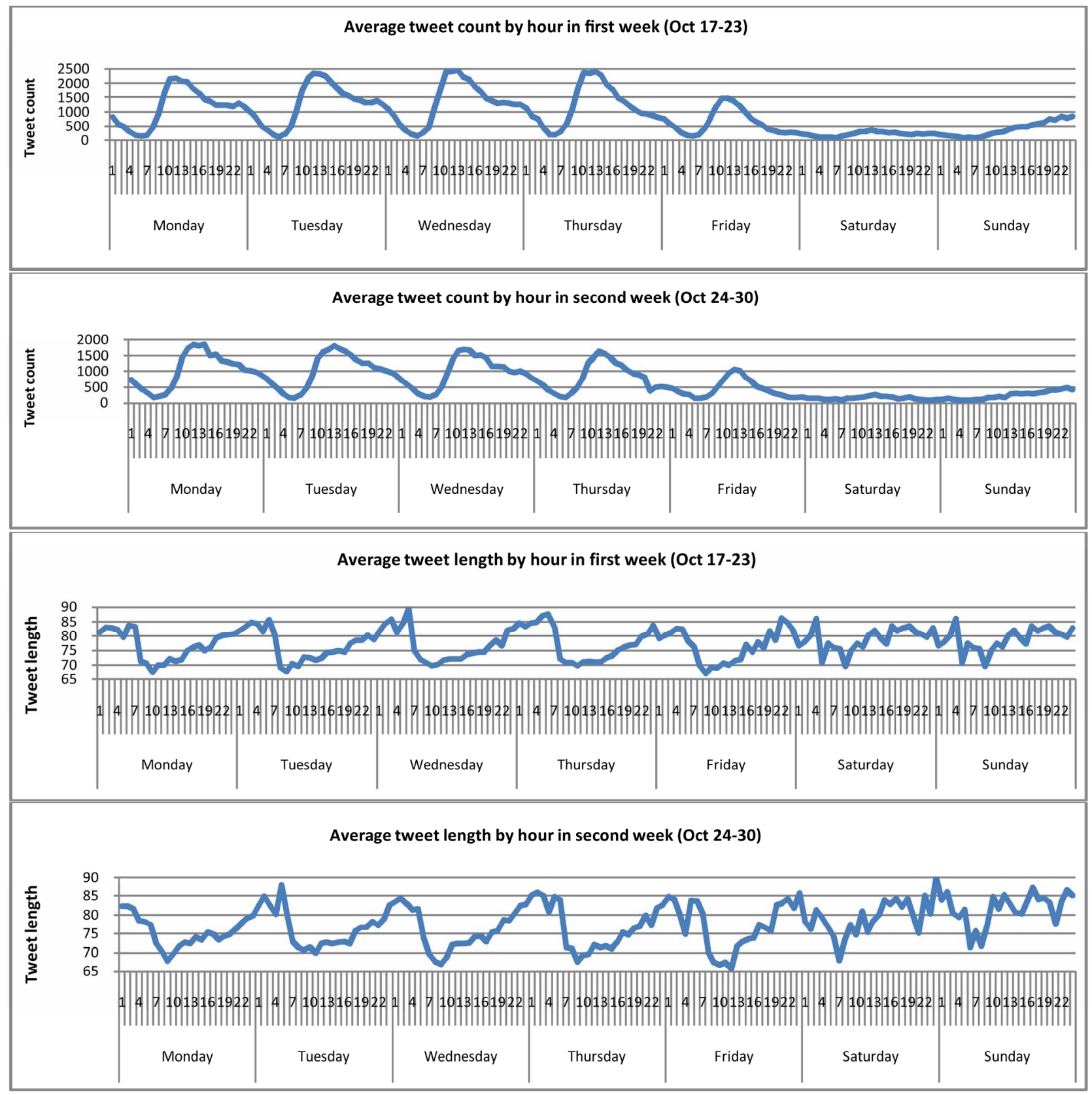

Figure 1.

Average tweet count and tweet length by hour in each week.

assgined -5 to each obscene word commonly used toward midterm exams. An opinion word combined with a negation word, such as "no" or "not", was assgined to its opposite weight. Each tweet was decomposed into $\mathrm{n}$ unique tokens (words and emoticons) and its sentiment score or value was defined as follows:

$$
\text { Sentiment }(\text { tweet })=\sum_{1}^{n} W_{i} C\left(T_{i}\right)
$$

where $W_{i}$ was the weight and $C\left(T_{i}\right)$ was the count of token $T_{i}$ in the tweet. According to this formula, positive sentiment 
values represent positive sentiment of a tweet, whereas negative sentiment values mean negative sentiment. Obviously, a zero value represents neutral sentiment.

The purpose of this study was to gauge the average Twitter sentiment on midterm exams. It was natural to solve our problem with a scoring system rather than a clssifier that predicts the sentiment as either positive, neutral, or negative. Since the scores are additive, three tweets with sentiment values $-3,0$, and 9 can have their average as 2, while the average sentiment is difficult to measure if these three tweets are classified as one negative, one neutral, and one positive.

To render the difference between a generic Twitter sentiment tool and our predictor, we ran Viralheat (http://www.viralheat.com) on Oct 17, 2011 using keyword midterm. Some of the sentiment predictions by Viralheat and our predictor are displayed in Table 2. Viralheat is designed to detect sentiment from general tweets, which contain opinons on a wide array of topics. It was not surprising that our sentiment values made more sense when looking at the actual text of each tweet. For example, the first tweet contained a happy emoticon and the word "happiest", but Viralheat gave negative $73 \%$, while our predictor gave a positive sentiment value of 6 .

\section{Results}

In the current study, two experiements were performed to assess the sentiment on midterm exams from a diverse group of students on Twitter. The first was determination of Twitter sentiment variation on midterms in real time by hour, day, and week during a period of two consecutive weeks. The time used here was Eastern Standard Time. The second was to investigate whether sentiment was assortative among the Twitter users who expressed their opinions on midterms.

\section{Real-Time Twitter Sentiment on Midterms}

Using the sentiment formula for a tweet introduced in Section 2, sentiment values were calculated for a stream of tweets on midterms collected from Oct 17 to Oct 30, 2011. These values were then sorted according to their time by hour, day, and week, and an average was taken for each hour (Figure 2). Our analysis suggested that the average sentiment of the first week (Oct 17-23) was more negative than the second week (Oct 24-30). As the midterm season approched to an end, Twitter users tended to be more hopeful about these exams and looked forward to college breaks and time for rest. The slope of increasing sentiment

Table 2.

Sentiments of several tweets on midterms evaluated by Viralheat and our predictor.

\begin{tabular}{lll}
\hline Tweet & Sentiment by Viralheat & Sentiment by our predictor \\
\hline $\begin{array}{l}\text { I am officially the happiest college student on earth. 98.5 on my chemo midterm and } \\
97 \text { on my calc midterm :))). }\end{array}$ & negative 73\% & 6 \\
$\begin{array}{l}\text { Blake got a 92\% on a math midterm he didn't even study for. Yes, that was third } \\
\text { person and you love it, don't you? }\end{array}$ & negative 99\% & -1 \\
$\begin{array}{l}\text { So I walk into class n theres a midterm I miss one class n of course it had to ve the one } \\
\text { where he announces the midterm }\end{array}$ & negative 99\% & 0 \\
$\begin{array}{l}\text { [UK] Longhorns football at “midterms”: AUSTIN—Consider this themid term assessment } \\
\text { at the halfwa... bit.ly/pYaZhH \#football \#UK }\end{array}$ & negative 99\% & 0 \\
$\begin{array}{l}\text { Back to this midterm study guide for tomorrow \#GrownFolksProblems } \\
\text { so far my midterm grades lookin good.need the rest of them to be put in }\end{array}$ & $\begin{array}{l}\text { negative } 97 \% \\
\text { negative } 97 \%\end{array}$ & 1 \\
\hline
\end{tabular}

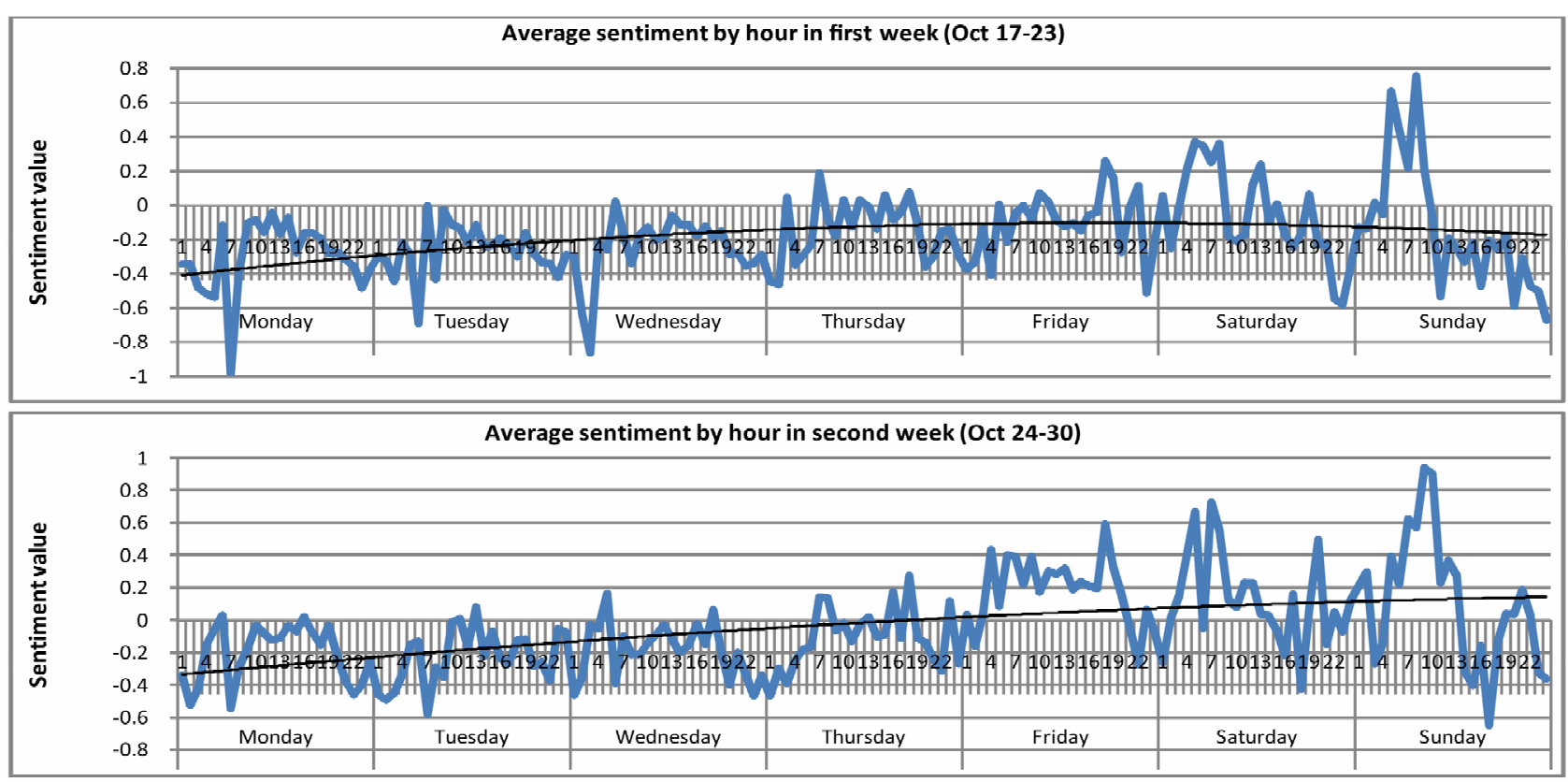

Figure 2.

Average hour-by-hour sentiment of each week. 
in the second week was larger than the first, which was caused by an apparent higher positive sentiment on Friday, Saturday, and Sunday in the second week.

There was a period during a weekday around the interval of 9:00 am to 5:00 pm that had maximum sentiment compared to rest of the day. However, Friday Oct 28 was one exception with high positive sentiment for an extended period of time. The sentiment values during a day at weekends usually reached their maximum between 5:00 am to 8:00 am, and then decreased after 8:00 am. The sentiment patterns during a weekday appeared different from a weekend.

A careful inspection of the collected tweets indicated that some of them contained obscene words toward midterms. To identify their usage in these tweets, we counted the average number of these words over the total number of tweets in each hour (Figure 3). The trend curves in Figure 3 implied that their usage decreased from Monday to Sunday, which was in the opposite trend of sentiment curves in Figure 2. It was evident that large number of these words would produce very negative sentiment. Although the peeks of the curves in Figure 3 occurred at a different time each day, but regularly around 7:00 am there was a local minimum usage of obscene words.

After looking into the dynamics of hourly and daily sentiment changes in Figure 2, we next examined the fluctuating patterns of sentiment between the first and second week. For this purpose, we counted the number of tweets according to their sentiment value by day, and stacked the counts of tweets of the same senitment from Monday to Sunday for each week (Figure 4). The Pearson correlation between the sentiment distributions of these two weeks is 0.99 , implying a high degree of similarity for the overall sentiment between the first and second week. Yet, our hour-by-hour sentiment curves in Figure 2 were able to show the varying nature of sentiment during these two weeks.

In addition to the sentiment evaluation, we also wanted to comprehend the readiness of this group of Twitter users for their midterms. The tweets that contained either the word "not ready" or "ready" were counted by day during a week and their ratio is presented in Figure 5. The first week had a mean of $18.85 \%$ and a standard deviation of 3.98 and second week had a mean of $18.57 \%$ and a standard deviation of 5.56 , which suggested the means of these ratios were similar between these two weeks. However, the ratio of tweets containing the word "not ready" vs. those containing "ready" was higher on Sunday, Monday, and Tuesday than other days in a week.

\section{Twitter Users Stratified by Their Sentiment on Midterms}

We explored the opportunity to detect group behavior of Twitter users according to their sentiment on midterms. Our hypothesis was that Twitter users with similar sentiment would tweet likewise. There are a few features used to describe each Twitter user that include, among others, the number of friends and followers. We sorted the number of friends and followers of each user according to his/her sentiment value by day and then took an average (Figure 6).

During the first week, Twitter users with negative sentiment had a mean of 241.35 and 265.25 for their average friends and followers respectively, while the positive had a mean of 293.16 and 324.74, suggesting that positive users usually had more friends and followers and as a result were more connected to others in online social media. Moreover, the Pearson correlation between the distributions of average number of friends and followers was 0.78 .

For the second week, Twitter users with negative sentiment had a mean of 227.83 and 261.63 for their average friends and followers respectively, whereas the positive had a mean of 278.58 and 350.16. Again, as in the first week, positive users had more friends and followers than negative users. The Pearson correlation between the distributions of average number of friends and followers was 0.97 , demonstrating stronger association in the second week than in the first. The high correlation value implied that the ratios of friends and followers of Twitter users of the same sentiment were similar, which is not true for general users.

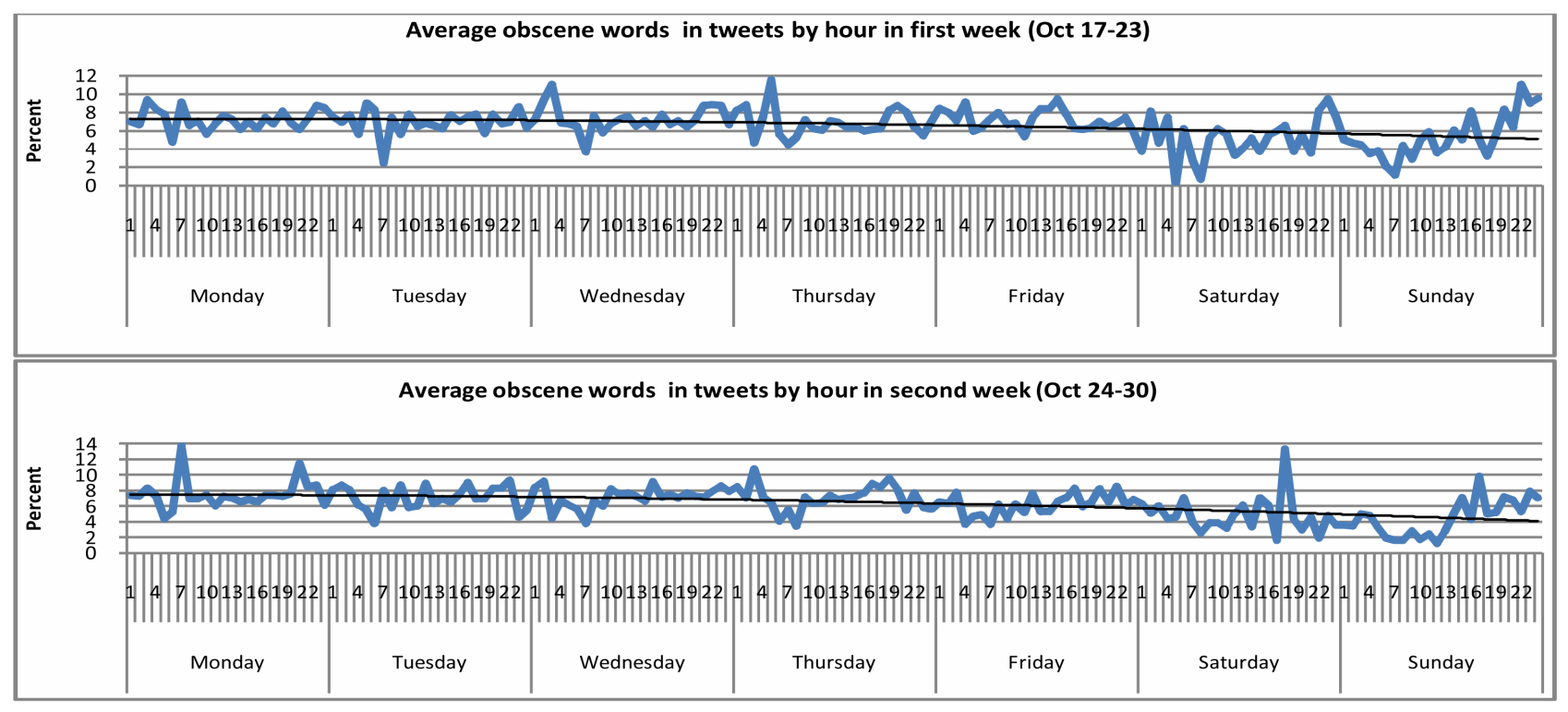

Figure 3.

Average obscene words in tweets by hour in each week. 


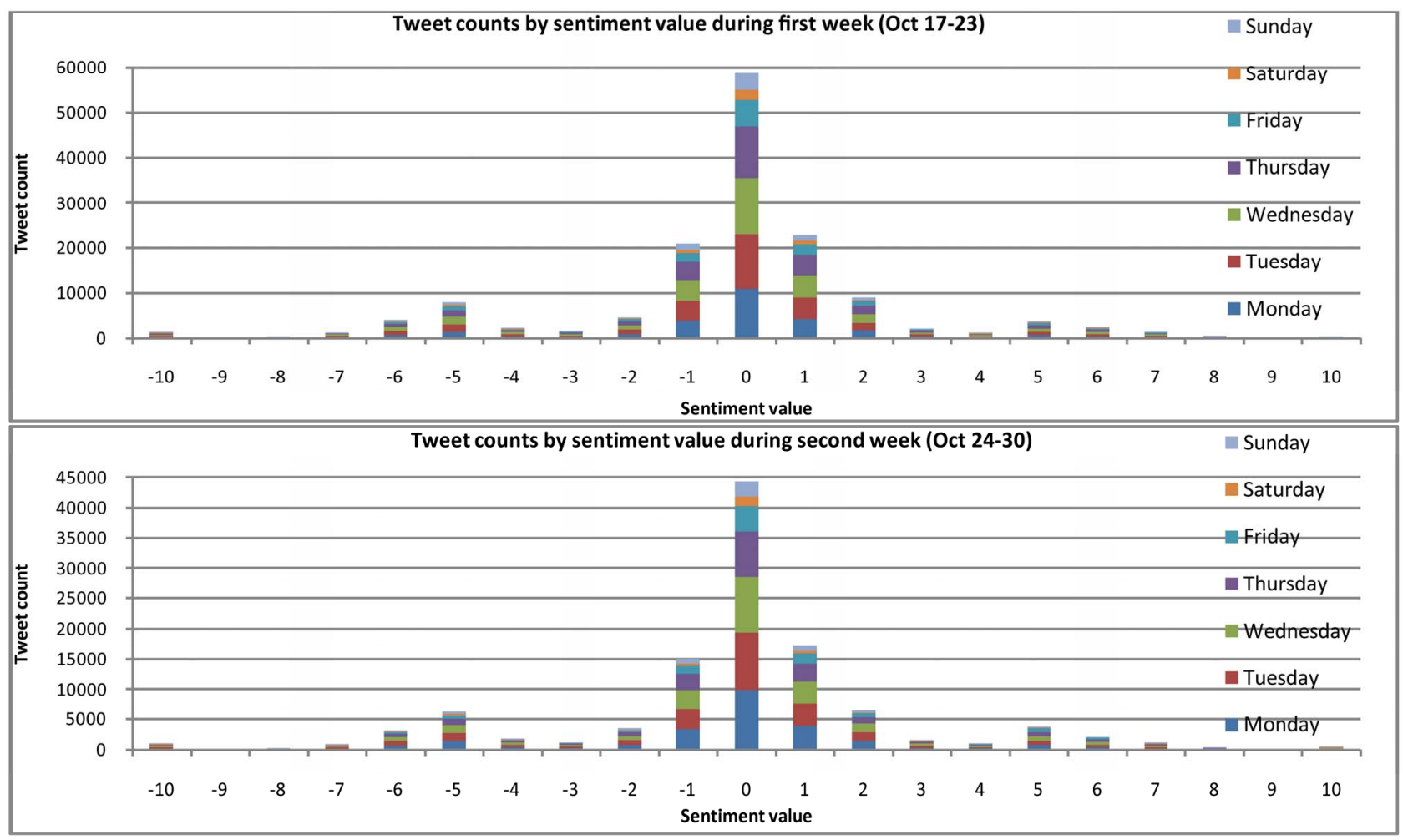

Figure 4.

Tweet counts according to their sentiment value stacked by day in each week.

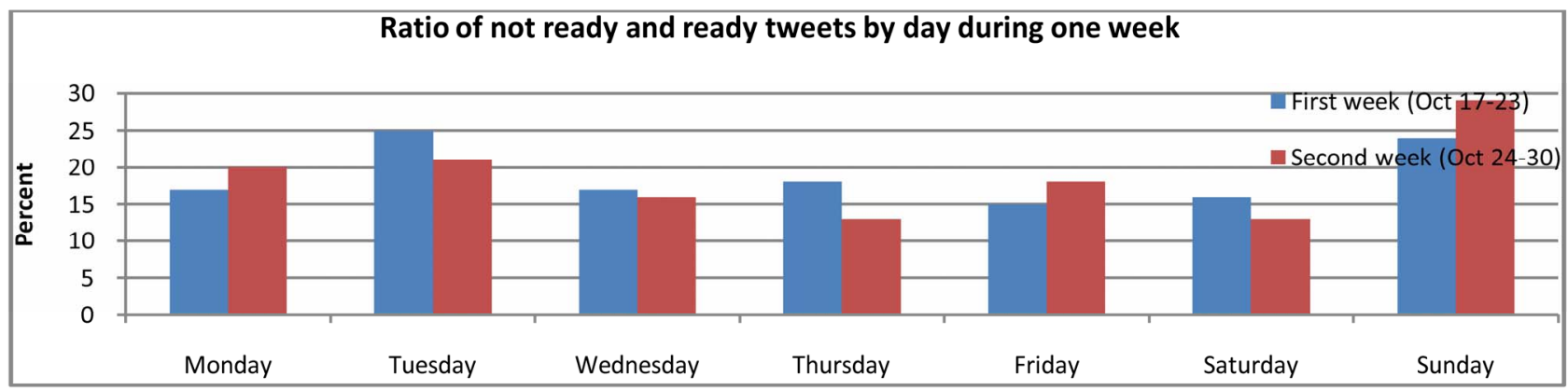

Figure 5.

Ratio of number of tweets containing word "not ready" vs. those containing "ready" in each week.

\section{Discussion and Conclusion}

Twitter is a fast-growing and massive repository of usergenerated content, which has been applied to influenza epidemics, political election, disaster mapping, and brand sentiment analysis. As the number of Twitters increases rapidly, mining their sentiment expressed in tweets is becoming an important research subject with great impact and potential.

Overtime Twitters have developed their own distinct expressions that often contain emoticons, slangs, and abbreviations, which evidently bring new challenges to the tradictional text mining techniques. The 140 character limit on tweets motivates Twitter users to be succinct and write their messages right on target. Because of these unique characters of Twitter messages, sentiment prediction of tweets requires special handlings. The current tools for Twitter sentiment are designed to detect general topics, therefore are not effective on a parcular topic since sentiment assessment is domain dependent.

In this report, we evaluated real-time sentiment of a stream of tweets on midterm exams collected for two consecutive weeks, from Oct 17 to Oct 30, 2011. Using an augmented opinion lexicon designed to tackle the specific characteristics of Twitter messages and the task at hand, a sentiment predictor was created. Supported by the results in (O’Connor et al., 2010), we believed that a sentiment predictor based a scoring system is more accurate to measure the average sentiment from this stream of tweets than a classifier that predicts tweets as positive, negative, or neutral sentiment, since our sentiment values are additive whereas the discrete labels of positive, negative and negative are not.

Analysis of this stream of tweets about the midterm exams by hour, day, and week illustrated the sentiment variation on this subject in real time. For both weeks, the overall trend curves of sentiment increased from Monday to Sunday. For 

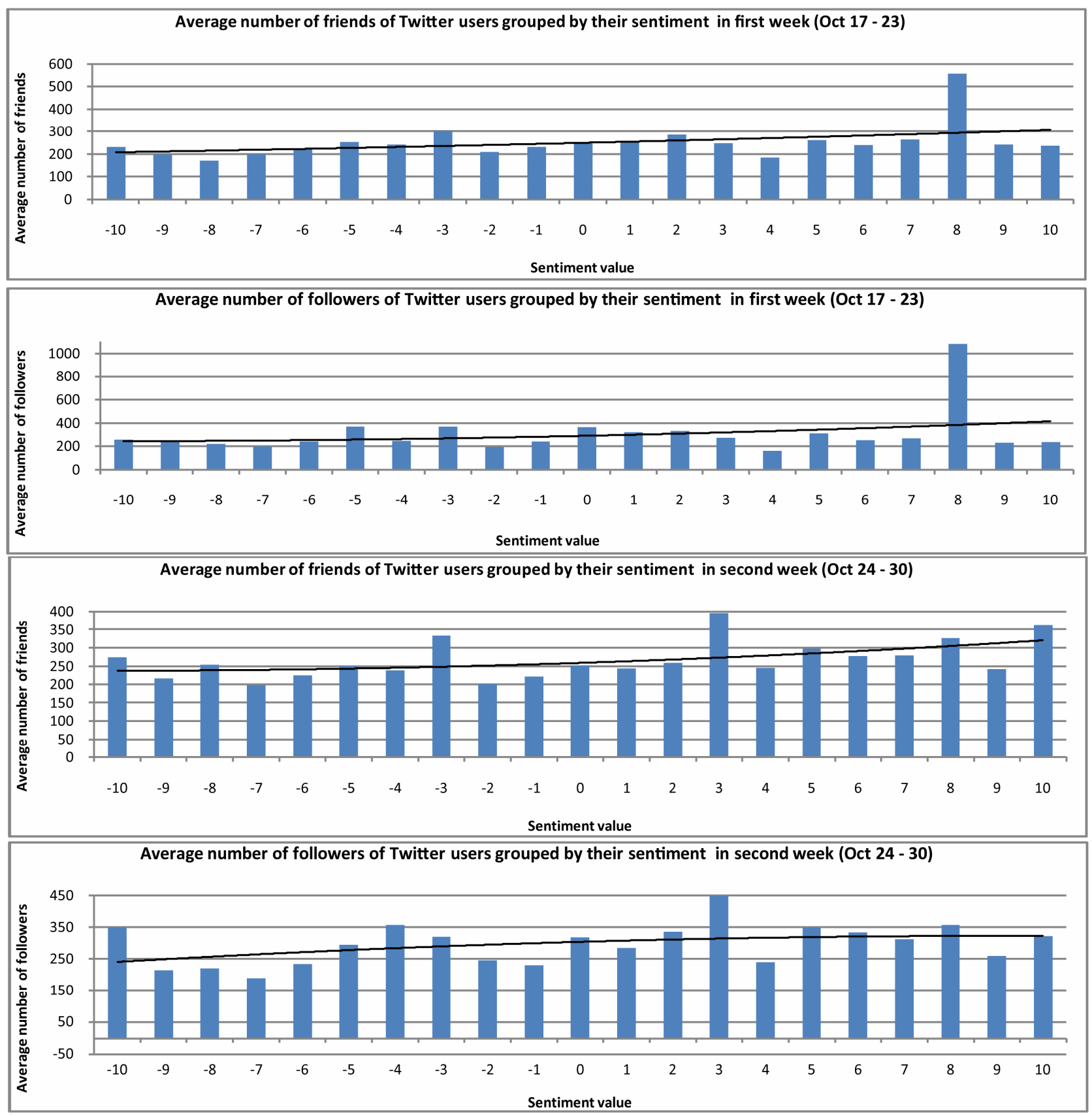

Figure 6.

Average number of friends and followers of Twitter users grouped by their sentiment in each week.

each weekday, there was a period around 9:00 am-5:00 pm EST that had maximum sentimet. On each weekend, the sentiment values during a day reached their maximum between 5:00 am to 8:00 am, and then decreased after 8:00 am. The Pearson correlation between the distributions of sentiment values stacked by day between the first and second week was 0.99, which indicated that the static characters of sentiment values of these two weeks were identical. However, our hour-by-hour sentiment detection was able to discover the changing nature of the sentiment on midterms.

Furthermore, we observed some consistent group behavior of Twitter users based on seemingly random behavior of each individual. The lowest number of tweets on midterms always occured around 5:00 am-6:00 am each day, and the maximum number was around 1:00pm except Sunday.

We also tested our hypothesis that Twitter users who expressed the same sentiment toward midterms would tweet in a similar fashion. Twitter users carrying positive sentiment seemed to have more friends and followers than negative users. The ratios of friends and followers of Twitter users with the same sentiment were close, which is not true for general users.

In summary, a stream of tweets on midterms from students on Twitter were collected using Twitter Stream API for two consecutive weeks. Real-time sentiment analysis on this tweet 
stream was conducted with an augemented lexicon based sentiment predictor. Our findings highlighted the dynamics of sentiment variation at various temporal granularity. Moreover, interesting group behavioral patterns of these student Twitters were uncovered from the random behavior of each individual.

\section{Acknowledgements}

We thank Houghton College for its financial support.

\section{REFERENCES}

Asur, S., \& Huberman, B. A. (2010). Predicting the future with social media. Proceedings of the ACM international conference on web intelligence, Toronto, 31 August-3 September 2010, 492-499.

Bollen, J., Pepe, A., \& Mao, H. N. (2011). Modeling public mood and emotion: Twitter sentiment and socio-economic phenomena. The International Conference on Weblogs and Social Media, Barcelona, 17-21 July 2011.

Go, A., Bhayani, R., \& Huang, L. (2009). Twitter sentiment classification using distant supervision. Stanford: CS224N Project Report.

Hu, M. Q., \& Liu, B. (2004). Mining and summarizing customer reviews. Proceedings of the ACM SIGKDD International Conference on Knowledge Discovery \& Data Mining, Seattle, 22-25 August 2004.

Jansen, B. J., Zhang, M., Sobel, K., \& Chowdury, A. (2009). Twitter power: Tweets as electronic word of mouth. Journal of the American Society for Information Science and Technology, 60, 2169-2188. doi:10.1002/asi.21149

Java, A., Song, X., Finin, T., \& Tseng B. (2007). Why we twitter: Understanding microblogging usage and communities. Proceedings of the 9th WebKDD and 1st SNA-KDD 2007 Workshop on Web mining and Social Network Analysis, San Jose, 12-15 August 2007, 56-65.

Krishnamurthy, B., Gill, P., \& Arlitt, M. (2008). A few chirps about twitter. Proceedings of the First Workshop on Online Social Networks, Seattle, 17-22 August 2008, 19-24.

Liu, B. (2010). Sentiment analysis and subjectivity, invited chapter for the handbook of natural language processing (2nd ed.). London/ Boca Raton: Chapman and Hall/CRC.

Lu, B., \& Tsou, B. K. (2010). Combining a large sentiment lexicon and machine learning for subjectivity classification. Proceedings of the International Conference of Machine Learning and Cybernetics, Qing- dao, 11-14 July 2010, 3311-3316.

MorNaaman, C.-H. L., \& Boase, J. (2010). Is it all about me? User content in social awareness streams. Proceedings of the 2010 ACM Conference on Computer Supported Cooperative Work, Savannah, 610 February 2010.

O’Connor, B., Balasubramanyan, R., Routledge, B. R., \& Smith, N. A. (2010). From tweets to polls: Linking text sentiment to public opinion time series. Proceedings of the International Artificial Intelligence Conference on Weblogs and Social Media, Atlanta, 11-15 July 2010, 122-129.

Pang, B., Lee, L., \& Vaithyanathan, S. (2002). Thumbs up? Sentiment classification using machine learning techniques. Proceedings of the Conference on Empirical Methods in Natural Language Processing, Philadelphia, 6-7 July 2002, 79-86.

Pang, B., \& Lee, L. (2008). Opinion mining and sentiment analysis. Foundations and Trends in Information Retrieval, 2, 1-135. doi:10.1561/1500000011

Pak, A., \& Paroubek, P. (2010). Twitter as a corpus for sentiment analysis and opinion mining. Proceedings of The International Conference on Language Resources and Evaluation Conference, Malta, 17-23 May 2010, 1320-1326.

Tan, S. B., Wang, Y. F., \& Cheng, X. Q. (2008). Combining learnbased and lexicon-based techniques for sentiment detection without using labeled examples. Proceedings of the 31st ACM SIGIR Conference on Research and Development in Information Retrieval, Singapore, 20-24 July 2008, 739-740.

Turney, P. (2002). Thumbs up or thumbs down? Semantic orientation applied to unsupervised classification of reviews. Proceedings of the Association for Computational Linguistics, Philadelphia, 6-12 July 2002, 417-424.

Velikovich, L., Blair-Goldensohn, S., Hannan, K., \& McDonald, R. (2010). The viability of web-derived polarity lexicons, human language technologies. The 2010 Annual Conference of the North American Chapter of the Association for Computational Linguistics, Association for Computational Linguistics, Los Angeles, 1-6 June 2010, 777785.

Vieweg, S., Hughes, A. L., Starbird, K., \& Palen, L. (2010). Microblogging during two natural hazards events: What twitter may contribute to situational awareness. Proceedings of the 28th International Conference on Human factors in Computing Systems, Atlanta, 10-15 April 2010, 1079-1088. 